2002

\title{
Evidence For Thermal Anisotropy Effects On Shear Modified Ion Acoustic Instabilities
}

\author{
E. E. Scime
}

A. M. Keesee

R. S. Spangler

M. E. Koepke

C. Teodorescu

See next page for additional authors

Follow this and additional works at: https://researchrepository.wvu.edu/faculty_publications

\section{Digital Commons Citation}

Scime, E. E.; Keesee, A. M.; Spangler, R. S.; Koepke, M. E.; Teodorescu, C.; and Reynolds, E. W., "Evidence For Thermal Anisotropy Effects On Shear Modified Ion Acoustic Instabilities" (2002). Faculty Scholarship. 528.

https://researchrepository.wvu.edu/faculty_publications/528 
Authors

E. E. Scime, A. M. Keesee, R. S. Spangler, M. E. Koepke, C. Teodorescu, and E. W. Reynolds 


\section{Evidence for Thermal Anisotropy Effects on Shear Modified Ion Acoustic Instabilities}

Earl E. Scime, Amy M. Keesee, Robert S. Spangler, and Mark E. Koepke, Catalin Teodorescu Department of Physics, West Virginia University, Morgantown, West Virginia 26506

Inclusion of thermal anisotropy effects is shown to be required to describe recently reported experimental measurements as shear-modified, ion-acoustic instabilities. For the reported experimental conditions, isotropic theory yields no instability growth that depends on the magnitude of the shear in the parallel flow. 
Recently, Teodorescu et al. presented detailed measurements of both parallel flow and parallel flow shear for both ions and electrons, parallel and perpendicular ion temperatures, electron temperature, growth rates, and wave numbers associated with low frequency, electrostatic instabilities in a Q-machine plasma. ${ }^{1}$ Although they suggested that their observations confirmed theoretical predictions for shear-modified ion acoustic waves, the comparison between theory and experiment was severely limited by the assumption of ion temperature isotropy in the model (ion temperature anisotropy was observed in the experiment). Specifically, in Figure 5 of Ref. 1, they presented experimental measurements of normalized instability growth rate versus normalized parallel flow shear that did not match theoretical predictions. In this Brief Communication, we use a model of shear-modified ion-acoustic instabilities that includes thermal anisotropy and present an alternative interpretation of their experimental measurements.

That parallel flow shear can significantly alter the thresholds, real frequencies, and growth rates of ion acoustic instabilities was demonstrated theoretically by Gavrishchaka et al.. ${ }^{2,3}$ In that work, the kinetic model developed by Ganguli et al. ${ }^{4}$ was extended to include the effects of parallel flow shear. It was then shown that currentdriven, ion acoustic instabilities could be excited by field aligned currents well below the critical values predicted by theories that did not include the effects of parallel flow shear. ${ }^{5}$ The theory calculations also suggested that such instabilities could be excited for ratios of ion to electron temperature of close to unity. In homogenous plasmas, nearly equal ion and electron temperatures completely suppress the growth of ion acoustic instabilities. ${ }^{5}$ In 2001, experimental evidence of ion acoustic instabilities in a Q-machine at sub-critical (according to previous theories) values of current was reported for plasmas with parallel flow shear. ${ }^{6}$ The more recent Teodorescu et al. measurements, in a different Q-machine plasma, included detailed measurements of all the relevant physical parameters necessary for quantitative comparison with theory. 
Because we have recently extended Gavrishchaka's work to include the effects of thermal anisotropy, ${ }^{7}$ we were curious if theoretical predictions for thermally anisotropic, shear-modified, ion-acoustic instabilities would be more consistent with the experimental data than the isotropic theory used by Teodorescu et al.. It is important to note that thermal anisotropy is not the only additional effect that can play an important role in these types of experiments. For example, Merlino has shown that simple radial density gradients can also significantly lower the current threshold for current-driven, electrostatic ion cyclotron instabilities. ${ }^{8}$

For a homogeneous plasma with thermal anisotropy and a parallel velocity shear, the kinetic dispersion relation, in normalized units, is given by ${ }^{7}$

$$
0=k^{2} \lambda_{D i \perp}^{2}+\widehat{T}_{i}+\sum_{n=-\infty}^{\infty} \Gamma_{n}(b) F_{n i}+\tau \widehat{T}_{e}\left(1+F_{0 e}\right)
$$

where

$$
\begin{gathered}
F_{n i}=\hat{T}_{i}^{1 / 2}\left[\frac{n\left(1-\hat{T}_{i}\right)+\hat{\omega} \hat{T}_{i}}{u \sqrt{2 b}}\right] Z\left(\frac{(\hat{\omega}-n) \hat{T}_{i}^{1 / 2}}{u \sqrt{2 b}}\right)-\frac{\hat{T}_{i} \hat{V}_{d}^{\prime}}{u}\left[1+\left(\frac{(\hat{\omega}-n)}{u \sqrt{2 b}} \hat{T}_{i}^{1 / 2}\right) Z\left(\frac{(\hat{\omega}-n)}{u \sqrt{2 b}} \hat{T}_{i}^{1 / 2}\right)\right], \\
F_{0 e}=(\xi) Z(\xi)+\frac{\hat{V}_{d}^{\prime}}{u \mu}[1+(\xi) Z(\xi)], \\
\xi=\sqrt{\frac{\tau \hat{T}_{e}}{2 \mu}}\left(\frac{\hat{\omega}}{u \sqrt{b}}-\widehat{V}_{d}\right),
\end{gathered}
$$

$\lambda_{D i \perp}^{2}=M_{i} v_{t h \perp i}^{2} / 4 \pi e^{2} n_{0}, \quad \hat{T}_{i} \equiv T_{i \perp} / T_{i \|}, \quad \Gamma_{m}(b) \equiv e^{-b} I_{m}(b), \quad b \equiv\left(k_{y} \rho_{i}\right)^{2}, \quad \rho_{i} \quad$ is the ion gyroradius, $\tau \equiv T_{\perp i} / T_{\perp e}, \hat{T}_{e} \equiv T_{e \perp} / T_{e \mid}, \hat{V}_{d} \equiv V_{d} / v_{i t h \perp}, \hat{V}_{d}^{\prime} \equiv\left(d V_{d} / d x\right) / \Omega, \Omega$ is the ion gyrofrequency, $V_{d}$ is the parallel (field-aligned) flow of electrons relative to ions, $\mathrm{v}_{t h \perp}$ is the thermal velocity in the perpendicular direction, $\mathrm{v}_{t h \|}$ is the thermal velocity in the parallel direction, $u \equiv k_{z} / k_{y}, \widehat{\omega} \equiv \omega / \Omega, n_{o}$ is the spatially uniform plasma density, and $\mu \equiv M_{i} / m_{e}$. In the derivation of Eq. (1), the weak shear limit, $\rho_{i} \ll L=V_{d} /\left(d V_{d} / d x\right)$, has been assumed. Note that Eq. (1) cleanly reduces to the isotropic theory for $T_{\perp}=T_{\|}$. The classic current-driven ion acoustic instability ${ }^{5}$ occurs at small values of $k_{y} \rho_{i}\left(k_{y} \rho_{i}\right.$ $<<1$ ). For a measured set of experimental parameters, Eq. (1) can be numerically solved 
to obtain the expected normalized, with respect to the ion gyrofrequency, growth rate and real frequency of the instability.

Shown in Fig. 1 as solid circles are the three normalized growth rate versus shear data points (labeled $a, b$, and $c)$ as reported in $\operatorname{Ref}[1]:\left(\hat{V}_{d}^{\prime}, u, \hat{V}_{d}, \tau, \hat{T}_{i}, b\right)=(-0.5,0.7$, $\left.15,1,1<\hat{T}_{i}<2, \sim 0.004\right),\left(-0.55,1.4,15<\hat{V}_{d}<300,1,1<\hat{T}_{i}<2, \sim 0.004\right),(-0.62,1.9$, $300,1,2.1, \sim 0.004)$. Note that the values for $\tau$ and $b$ were not explicitly given in Ref [1], but were deduced from comments in the text and elsewhere. ${ }^{9}$ If isotropic ions are assumed and these experimental parameters used in Eq. (1), we find that in no case is a positive normalized growth rate obtained: $\hat{\gamma}_{a}=-0.026, \hat{\gamma}_{b}=-0.027, \hat{\gamma}_{c}=-0.039$. Note that for the experimental parameters corresponding to point $b$, the maximum possible drift, $\hat{V}_{d}=300$, of electrons in the ion frame as reported in Ref [1] was used. Thus, thermally isotropic theory predicts no growth of shear-modified ion-acoustic instabilities for any of the reported parameters of the Teodorescu et al. experiments. In the case of isotropic ions, positive growth rates are obtained from the theory only for much smaller values of inverse propagation angle, ${ }^{10} u \sim 0.1$, with simultaneously larger values of normalized shear, $\hat{V}_{d}^{\prime} \sim-1.5$ (as were reported in the Agrimson et al. experiments ${ }^{6}$ where $\widehat{V}_{d} \sim 7$ and $\left.b \sim 0.02\right)$.

Also shown in Figure 1 as open squares are the predicted normalized growth rates if the measured thermal anisotropies of the ions are used in the calculations, $\hat{\gamma}_{a}=-0.017$, $\hat{\gamma}_{b}=0.003, \hat{\gamma}_{c}=0.009 .{ }^{1,9}$ Note that the growth rate for the first set of experimental parameters is still negative, $\hat{\gamma}_{a}=-0.016$. This indicates that if the measured waves are ion acoustic instabilities and the model assumptions match the experimental conditions, parallel flow shear and thermal anisotropy are not destabilizing enough to yield positive growth at such large relative ion to electron temperatures, i.e., $\tau \sim 1$, and such small relative flows, $\hat{V}_{d}=15$. Careful analysis of the numerical calculations does indicate that shear modified, ion acoustic instabilities can be unstable at the experimental parameters corresponding to the first data point, but only at much smaller values of $u$, i.e., for nearly 
perpendicular propagation of the waves $(u \sim 0.1)$. Even if the measured parallel wave number $(u=0.7)$ was at the lower end of the range of experimental uncertainty $( \pm 15 \%)$, the theory would still yield a negative normalized growth rate.

For the other two experimental conditions, positive growth rates are obtained only if thermal anisotropy is included. If the normalized shear is set to zero in the calculations, $\hat{V}_{d}^{\prime}=0$, negative growth rates are obtained for all three set of experimental conditions. Thus, both parallel flow shear and thermal anisotropy are required to obtain positive growth rates in the theory for points $b$ and $c$. As an aside, we note that the theoretical predicted, normalized real frequencies for thermally anisotropic, shear-modified, ion acoustic instabilities at the Teodorescu et al. experimental parameters are $\widehat{\omega}_{a}=0.09, \widehat{\omega}_{b}$ $=0.15$, and $\widehat{\omega}_{c}=0.19$. Such real frequency values compare favorably with the experimentally observed frequencies. ${ }^{9}$

Qualitative agreement between theory and experiment (at least for the $b$ and $c$. experiment parameters) always encourages belief in the appropriateness of the theoretical model. However, we feel compelled to note the following caveats. First, the available measurements are sparse and more than three data points would provide a better test of the theoretical predictions. Second, the theory is a fundamentally local theory, i.e., the ion gyroradius, $\rho_{i}$, must be smaller than both the shear scale, $L=V /(d V / d x)$, and the spatial extent of the shear region. For the experimental parameters reported in Ref [1], $\rho \sim 0.5$ $\mathrm{cm}, L \sim 2.5 \mathrm{~cm}$, and the shear region extends over approximately $0.75 \mathrm{~cm}$ (according to Fig. 2 in Ref [1]). Ions with gyroradii of $0.5 \mathrm{~cm}$ or greater spend a significant fraction of time outside of the shear region. Thus, a fully non-local, thermally anisotropic, theory is required for comparison with the experimental data. The role of density gradients also warrants additional study given that parallel flow driven ion cyclotron waves have been found to be destabilized by density gradients. ${ }^{8}$

Third, comparison of experimental data to the predicted zeroth order shifts in the ion acoustic wave phase velocity and frequency ${ }^{7}$, as is done in Ref. [1], requires that 
$\left(k_{y} \rho\right)^{2}<<1$. For $\rho \sim 0.5 \mathrm{~cm}$ and the experimentally measured value of $k_{y} \sim 0.56 \mathrm{~cm}^{-1}$, this constraint is satisfied. ${ }^{1}$ For $\rho \sim 0.5 \mathrm{~cm}$ and the experimentally measured value of $k_{y} \sim$ $1.75 \mathrm{~cm}^{-1},\left(k_{y} \rho\right)^{2} \sim 1$ and care must be taken to include all of the significant higher harmonic terms in the theoretical calculations. ${ }^{7}$ Fourth, we note that cylindrical normalmode geometry forces the measured azimuthal wave number range $\left(k_{y} \sim 0.56-1.75\right.$ $\mathrm{cm}^{-1}$ ) to correspond to a cylindrical region $1.1-3.5 \mathrm{~cm}$ in diameter, well within the Qmachine plasma column but outside of the $r \sim 0.4 \mathrm{~cm}$ shear region. One possible cause of the extended radial region of finite wave amplitude might be the lack of cylindrical symmetry in the parallel flow shear.

In summary, we have shown that both parallel shear and ion temperature anisotropy are required for the growth of shear-modified, ion-acoustic waves at two of three experimental parameters. The lack of positive instability growth for the first set of experimental parameters is not yet understood but could be explained by differences between the experimental geometry and the assumptions in the model.

\section{ACKNOWLEDGMENTS}

This work was performed with support from National Science Foundation grants ATM-99988450 and ATM-9816261, the Office of Naval Research, the Naval Research Laboratory (NRL), and the U.S. Department of Energy Fusion Science Graduate Fellowship Program. 


\section{References}

${ }^{1}$ C. Teodorescu, E.W. Reynolds, and M.E. Koepke, Phys. Rev. Lett. 88, 185003 (2002).

${ }^{2}$ V. V. Gavrishchaka, G. I. Ganguli, S. B. Ganguli, Phys. Rev. Lett. 80, 728 (1998).

${ }^{3}$ V. V. Gavrishchaka, S. B. Ganguli, G. I. Ganguli, J. Geophys. Res. 104, 12,683 (1999).

${ }^{4}$ G. Ganguli, M. J. Keskinen, H. Romero, R. Heelis, T. Moore, C. Pollock, J. Geophys. Res. 99, 8873 (1994); G. Ganguli, Y. C. Lee, P. J. Palmadesso, Phys. Fluids 31, 823 (1988).

5 J. M. Kindel and C.F. Kennel, J. Geophys. Res. 76, 3055 (1971).

${ }^{6}$ E. Agrimson, N. D’Angelo, R. L. Merlino, Phys. Rev. Lett. 86, 5282 (2001).

${ }^{7}$ R. Spangler, E. Scime, and G. Ganguli, Phys. Plasmas 9, 2526 (2002).

${ }^{8}$ R. L. Merlino, Phys. Plasmas 9, 1824 (2002).

${ }^{9}$ C. Teodorescu, M.E. Koepke, and E.W. Reynolds, J. Geophys. Res., submitted (2002).

${ }^{10}$ A typographical error in the theory parameters given in the caption of Fig. 5 of Ref. 1 $(u=0.7,1.4,1.9)$ has been corrected to $(u=0.11,0.12,0.12)$ in Ref. 9. 


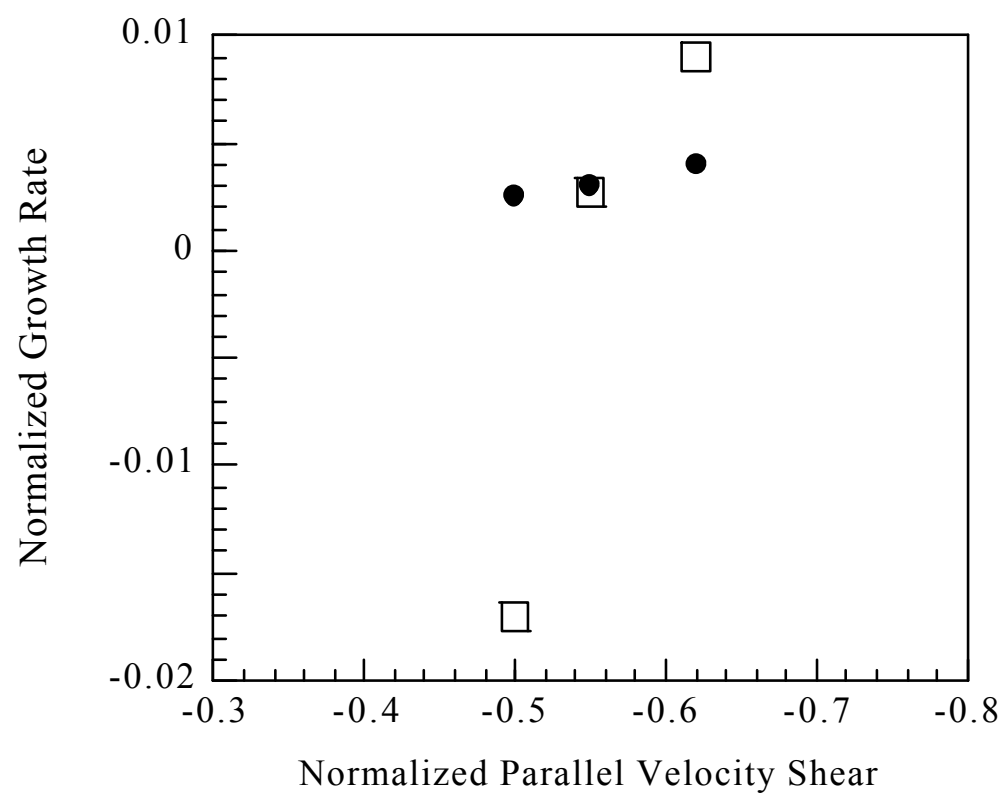

Figure 1. ( ) Experimentally measured growth rate as a function of parallel flow shear for $\left(\hat{V}_{d}^{\prime}, u, \widehat{V}_{d}, \tau, \hat{T}_{i}, b\right)=\left(-0.5,0.7,15,1,1<\hat{T}_{i}<2, \sim 0.004\right),(-0.55,1.4,15<u<300$, $\left.\left.1,1<\hat{T}_{i}<2, \sim 0.004\right),(-0.62,1.9,300,1,2.1, \sim 0.004) .{ }^{1} \square\right)$ Predicted growth rate for thermally anisotropic, shear-modified, ion-acoustic instabilities for $\left(\hat{V}_{d}^{\prime}, u, \widehat{V}_{d}, \tau, \hat{T}_{i}, b\right)$ $=(-0.5,0.7,15,1,1.4,0.004),(-0.55,1.4,300,1,1.8,0.004),(-0.62,1.9,300,1,2.1$, $0.004)$. 